\title{
Spectroscopic ellipsometry on thin titanium oxide layers grown on titanium by plasma oxidation
}

G. Droulers, A. Beaumont, J. Beauvais, and D. Drouin

Citation: Journal of Vacuum Science \& Technology B, Nanotechnology and Microelectronics: Materials, Processing, Measurement, and Phenomena 29, 021010 (2011); doi: 10.1116/1.3553209

View online: http://dx.doi.org/10.1116/1.3553209

View Table of Contents: http://avs.scitation.org/toc/jvb/29/2

Published by the American Vacuum Society

Contact Hiden Analytical for further details: W www.HidenAnalytical.com E info@hiden.co.uk

CLICK TO VIEW our product catalogue

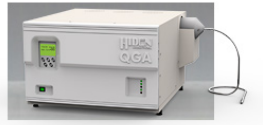

Gas Analysis

dynamic measurement of reaction gas streams catalysis and thermal analysis

molecular beam studies

dissolved species probes

fermentation, environmental and ecological studies

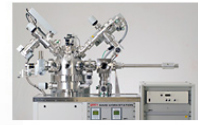

Surface Science , UHVTPD

SIMS

end point detection in ion beam etch elemental imaging - surface mappin

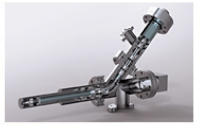

Plasma Diagnostics plasma source characterization etch and deposition process reaction , kinetic studies analysis of neutral and radical species

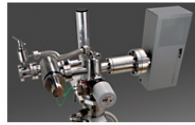

Vacuum Analysis - partial pressure measurement and control of process gases ' reactive sputter process contro , vacuum diagnostics vacuum coating process monitoring 


\title{
Spectroscopic ellipsometry on thin titanium oxide layers grown on titanium by plasma oxidation
}

\author{
G. Droulers, ${ }^{\text {a) }}$ A. Beaumont, J. Beauvais, and D. Drouin \\ Nanofabrication and Nanocharacterization Research Center, Universite de Sherbrooke, Sherbrooke, Quebec \\ JIK 2R1, Canada
}

(Received 13 September 2010; accepted 19 January 2011; published 4 February 2011)

\begin{abstract}
Electronic devices based on tunnel junctions require tools able to accurately control the thickness of thin metal and oxide layers on the order of the nanometer. This article shows that multisample ellipsometry is an accurate method to reach this goal on plain uniform layers, in particular for titanium. From these measurements, the authors carefully studied the oxidation rate of titanium thin films in an oxygen plasma. The authors found that the oxide thickness saturates at $5.4 \pm 0.4 \mathrm{~nm}$ after $10 \mathrm{~min}$ in the plasma with an ion acceleration power of $30 \mathrm{~W}$. Increasing this power to $240 \mathrm{~W}$ increases the saturation value to $7.6 \pm 0.4 \mathrm{~nm}$. An x-ray photoelectron spectroscopy study of the oxide has shown that the oxide created by $\mathrm{O}_{2}$ plasma is stoichiometric $\left(\mathrm{TiO}_{2}\right)$. The developed model was also used to measure the thicknesses of titanium and titanium oxide layers that have been polished using a chemical mechanical planarization process and a material removal rate of 5.9 $\mathrm{nm} / \mathrm{min}$ is found with our planarization parameters. (c) 2011 American Vacuum Society.
\end{abstract}

[DOI: $10.1116 / 1.3553209]$

\section{INTRODUCTION}

Progress in microfabrication and unique properties of tunnel junctions have led many research groups to study tunneling devices and propose them as candidates for a wide range of logic and analog applications. Specific properties of semiconductors are sometimes mandatory for the operation of these devices (e.g., resonant tunneling diodes ${ }^{1}$ and metalinsulator tunneling transistors ${ }^{2,3}$ ). Other features of some metals are also exploited in tunneling devices: ferromagnetism in magnetic random access memories ${ }^{4,5}$ and magnetic tunneling transistors ${ }^{6,7}$ and superconductivity in rapid single flux quantum devices ${ }^{8}$ and superconducting quantum interference devices. ${ }^{9}$ Moreover, the high density of carriers in metals improves the performances of single electron transistors (SETs). ${ }^{10}$ Whatever the fabrication process used, tunnel junctions are elaborated whether by insulator deposition onto the metal or by oxidation of the metal itself. While the former potentially faces issues related to diffusion and defects at the metal/insulator interface, ${ }^{11}$ the latter requires accurate characterization of thickness and electrical properties since it may vary significantly with oxidation parameters. This thickness characterization is conveniently performed on unstructured thin films, commonly by spectroscopic ellipsometry. However, this method is known to be difficult to handle with thin metallic films because they are optically absorbent and often discontinuous. ${ }^{12,13}$ The present article shows that this difficulty may be lifted by fabricating several samples sharing the same metallic film but oxidized with different parameters. Indeed, when the data set is fit with appropriate models and a multisample method, the thickness of an insulator grown on the same metal with any parameter may be unambiguously determined by adding it to the refer-

${ }^{a)}$ Electronic mail: gabriel.droulers@ usherbrooke.ca ence database and refitting the data set. This is illustrated by the demonstration of thin insulators grown at the surface of Ti layers by $\mathrm{O}_{2}$ plasma oxidation. This example is of practical interest because it is a critical step for the fabrication of room-temperature single electron transistors with a process called nanodamascene. ${ }^{14,15}$ It is also shown that multisample fitting of ellipsometric data enables one to characterize the thickness of titanium layers even when it is decreased by chemical mechanical planarization (CMP).

\section{MATERIALS AND METHODS}

Two series of samples were fabricated. The first, hereafter referred to as series A, was prepared as follows: first, on a silicon wafer (100 oriented) an 89-nm-thick dry thermal $\mathrm{SiO}_{2}$ layer was grown at $1050{ }^{\circ} \mathrm{C}$ for $77 \mathrm{~min}$ in a $60 \% \mathrm{~N}_{2}$ and $40 \% \mathrm{O}_{2}$ ambient atmosphere followed by an electron beam evaporation, with a rate of $0.2 \mathrm{~nm} / \mathrm{s}$, of $25-\mathrm{nm}$-thick Ti layers. Series B was oxidized for $80 \mathrm{~min}$ in the same furnace conditions for a $\mathrm{SiO}_{2}$ thickness of $100 \mathrm{~nm}$ and had evaporated Ti layers of 15, 20, and $25 \mathrm{~nm}$ thicknesses. The samples of series A were then oxidized in a pure $\mathrm{O}_{2}$ plasma carried out in a PLASMALINE 211 barrel asher at roomtemperature modulo, the local heating routinely observed in such a desktop barrel asher. The power used ranged from 30 up to $125 \mathrm{~W}$ and oxidation time was varied from $30 \mathrm{~s}$ up to $60 \mathrm{~min}$. Series B was oxidized in a surface technology systems multiplex inductively coupled plasma (ICP) standard rate III-V system using an $\mathrm{O}_{2}$ plasma with $600 \mathrm{~W}$ of coil power, $30-240 \mathrm{~W}$ of platen power, and times ranging between $30 \mathrm{~s}$ and $10 \mathrm{~min}$. The pressure in the chamber was set to $40 \mathrm{mTorr}$.

Spectroscopic ellipsometry was carried out in an alpha-SE ellipsometer (J.A. Woollam Co., Inc.) at a $70^{\circ}$ angle of incidence. The wavelengths ranged from 380 to $900 \mathrm{~nm}$ and the data were fit with COMPLETEEASE software (J.A. Woollam 
Co., Inc.) by minimization of the mean square error (MSE) which is given by

$$
\begin{aligned}
\text { MSE }= & 1000\left\{\frac { 1 } { 3 n - m } \sum _ { i = 1 } ^ { n } \left[\left(N_{E_{i}}-N_{G_{i}}\right)^{2}+\left(C_{E_{i}}-C_{G_{i}}\right)^{2}\right.\right. \\
& \left.\left.+\left(S_{E_{i}}-S_{G_{i}}\right)^{2}\right]\right\}^{1 / 2},
\end{aligned}
$$

where $n$ is the number of wavelengths, $m$ is the number of fit parameters, and $N, C$, and $S$ are the experimental (with the subscript $E$ ) and model generated (with the subscript $G$ ) data. ${ }^{16}$ The $N, C$, and $S$ values are defined in terms of traditional psi-delta notation as $N=\cos (2 \Psi), C=\sin (2 \Psi) \cos (\Delta)$, and $S=\sin (2 \Psi) \sin (\Delta)$. The first term is a normalization prefactor enabling the MSE to be equal to unity in the case of an ideal fit assuming typical precision and accuracy of the measurement. Indeed, the best error that is susceptible to be reached with our apparatus is around $0.001,{ }^{16}$ which explains the choice of a normalization factor of 1000, leading to an ideal MSE value of 1 . All best-matched results were always visually inspected to avoid nonphysical features. Equation (1) is an unbiased error function which was chosen because our goal was to bring simulated psi-delta curves as close to every experimental data point as possible. A biased error function might also have been used, but this would have required weighing the error on each data point according to the optical properties of the materials studied at the related wavelength. However, in our case the exact properties of $\mathrm{TiO}_{2}$ were not known a priori, explaining the choice of an unbiased error function.

Profilometry measurements were carried out on a Dektak 150 Profilometer with a $12.5 \mu \mathrm{m}$ stylus radius and $5 \mathrm{mg}$ of force applied on it. CMP was performed with an ULTRATEC Multipol CMP system, using a $0.05 \mu \mathrm{m}$ colloidal silica slurry, with a pressure of $280 \mathrm{~g} / \mathrm{cm}^{2}$ and a rotation speed of $15 \mathrm{rpm}$. X-ray photoelectron spectroscopy (XPS) surface analysis was performed using a Kratos Axis Ultra DLD spectrometer employing an Al monochromatic anode (1486.6 $\mathrm{eV})$. The $\mathrm{x}$-ray source was operated at $15 \mathrm{kV}$ and $15 \mathrm{~mA}$. The analyzer pass energy was set at $20 \mathrm{eV}$. Charge neutralization current was applied and curves were calibrated using the $\mathrm{C} 1 s$ peak at $285 \mathrm{eV}$ as in Ref. 17.

\section{ELLIPSOMETRIC MODEL}

As is well known, ellipsometric measurements are relatively easy to obtain. However, in order to extract useful parameters such as dielectric function and thickness of layers, one must use a theoretical model of the measurement and find the best match between theory and experiment. To do this, a sample model can be constructed of different layer models reproducing the optical behavior of each layer. There are two different types of layer models that can be used to fit spectroscopic ellipsometry data. The first one is based on a tabulated set of data for the index of refraction $n$ and the extinction coefficient $k$. This type of layer model is often based on published experimental measurements and gener- ally works well on pure and highly crystalline materials such as semiconductor substrates. The very small number of fit parameters (actually only thickness) reduces the possibility of multiple solutions for the fit parameters, this being called parameter correlation. On the other hand, mathematical dispersion models propose to carry out Lorentz, Sellmeier, Cauchy, and many other layer models to simulate the dielectric function of materials. ${ }^{13}$ They are useful when dealing with materials for which no $n$ and $k$ values are reported, which is commonly the case for melted, nonideal, or unknown materials. However, since there are more parameters to fit in this type of layer model, the possibility of correlation is greater.

The layer model used for the substrate is a tabulated set of data for semiconductor grade silicon single crystal, originally published by Herzinger et al. ${ }^{18}$ For the $\mathrm{SiO}_{2}$ layer, a Sellmeier layer model was chosen since it provided the lowest MSE values when fitting spectra obtained on reference thermal $\mathrm{SiO}_{2}$ samples. It is a mathematical dispersion model, given by Eq. (2),

$$
n=\left(\varepsilon_{\infty}+\frac{A \lambda^{2}}{\lambda^{2}-B^{2}}-E \lambda^{2}\right)^{1 / 2}
$$

It is based on the transparent region $(k \rightarrow 0)$ of a Lorentz classical model ${ }^{13,16}$ and is well adapted for films that are transparent in the measurement wavelength range. The layer model used for the $\mathrm{TiO}_{2}$ layer is a Cauchy model. This is also a mathematical dispersion model [see Eq. (3)] obtained from a series expansion of the Sellmeier layer model and it is therefore an approximate function of it, ${ }^{13}$

$$
n=A+\frac{B}{\lambda^{2}}+\frac{C}{\lambda^{4}}
$$

This layer model was assumed to have $k=0$ and was chosen because it resulted in lower MSE values than with the Sellmeier layer model although they both should be similar in the studied wavelength range. Moreover, thicknesses measured with this layer model were found consistent to the profilometry measurements presented further. It is more challenging to characterize titanium since it absorbs light in all the wavelengths used by our ellipsometer. This means that the interference from the underlying $\mathrm{SiO}_{2}$ layer is dependent on the thickness of the absorbing Ti which appears in data as a change in dielectric function leading to parameter correlation. Multisample analysis (MSA) can be used to reduce the degree of correlation ${ }^{12}$ (as detailed in the next paragraph). As mentioned by Fujiwara, ${ }^{13}$ the dielectric function of an absorbing film can be modeled by the combination of a number of Lorentz oscillators accounting for the bound electron response to the incident light. In this study, three oscillators were needed in order to get a good match with the data [Eq. (4)], 
TABLE I. Best-fit parameters for the ellipsometric model of $\mathrm{TiO}_{2} / \mathrm{Ti} / \mathrm{SiO}_{2} / \mathrm{Si}$. Parameters are defined according to Eqs. (2)-(4). The two sets of values for the $\mathrm{TiO}_{2}$ layer are for the native oxide and for the oxide created in the $\mathrm{O}_{2}$ plasma.

\begin{tabular}{lccc}
\hline \hline $\mathrm{TiO}_{2}$ layer (Cauchy) & Native & Plasma & \\
\hline$A$ & 2.27 & 2.02 & \\
$B$ & 0.51 & 0.21 & \\
$C$ & 0.036 & 0.008 & \\
& & & \\
$\mathrm{Ti}$ layer (Lorentz) & Oscillator 1 & Oscillator 2 & Oscillator 3 \\
\hline Index offset $\left(\varepsilon_{\infty}\right)$ & 0.034 & 0.034 & 0.034 \\
Amplitude $(\mathrm{Amp})$ & 213 & 12.9 & 19.6 \\
Broadening $(\mathrm{Br})$ & 7.23 & 1.49 & 2.01 \\
Energy (En) & 0 & 1.69 & 3.02 \\
& & & \\
SiO ${ }_{2}$ layer $(\mathrm{Sellmeier})$ & Thermal & & \\
\hline Index offset $\left(\varepsilon_{\infty}\right)$ & 0 & & \\
Amplitude $(A)$ & 2.14 & & \\
Center energy $(B)$ & 0.055 & & \\
IR pole amplitude $(E)$ & 0.018 & & \\
\hline \hline
\end{tabular}

$$
\varepsilon_{2}=\operatorname{Einf}+\sum_{i=1}^{3} \frac{\mathrm{Amp}_{n} \mathrm{Br}_{n} \mathrm{En}_{n}}{\operatorname{En}_{n}^{2}-E^{2}-i E \mathrm{Br}_{n}} .
$$

Since the Lorentz model is Kramers-Kronig consistent, the software uses this feature to integrate the best-match model of $\varepsilon_{2}$ (imaginary part) to find $\varepsilon_{1}$ (real part). The best fit parameters of the three models are shown in Table I.

MSA is a good method for reducing the number of fit parameters by providing more information on the samples. ${ }^{12}$ MSA works by assuming one set of optical functions for all samples and by letting selected parameters vary from sample to sample. For MSA to give appropriate results, it is important that the samples used are presumably similar in their dielectric functions and layer physical properties, namely roughness among the set. In the present MSA study, only thickness of the $\mathrm{Ti}$ and $\mathrm{TiO}_{2}$ was varied between samples. Note that in the case of the CMP fits, $\mathrm{SiO}_{2}$ layer thickness was added as a fit parameter since it changed during the process. To reduce the correlation, MSA has been used on many samples before the $\mathrm{Ti}$ evaporation fabrication step. With the information obtained from these early measurements, all parameters of the $\mathrm{SiO}_{2}$ layer (Sellmeier's index offset, amplitude, center energy, and IR pole amplitude) were set to fixed values in the following analysis. The same was done on a series of samples with the same Ti thickness (evaporation) unintentionally oxidized in air to fix the parameters of the $\mathrm{TiO}_{2}$ layer (Cauchy's $A, B$, and $C$ parameters) and certain parameters of the $\mathrm{Ti}$ layer as well (Lorentz's Br, En, and Einf). These three parameters of the Lorentz oscillator were fixed because they had relatively small effects on the quality of the fit for different thicknesses. Fixing them also considerably reduced the correlation in the sample model. Since the optical functions of thin absorbing films vary with thickness, ${ }^{12,13,19}$ simultaneous regression of

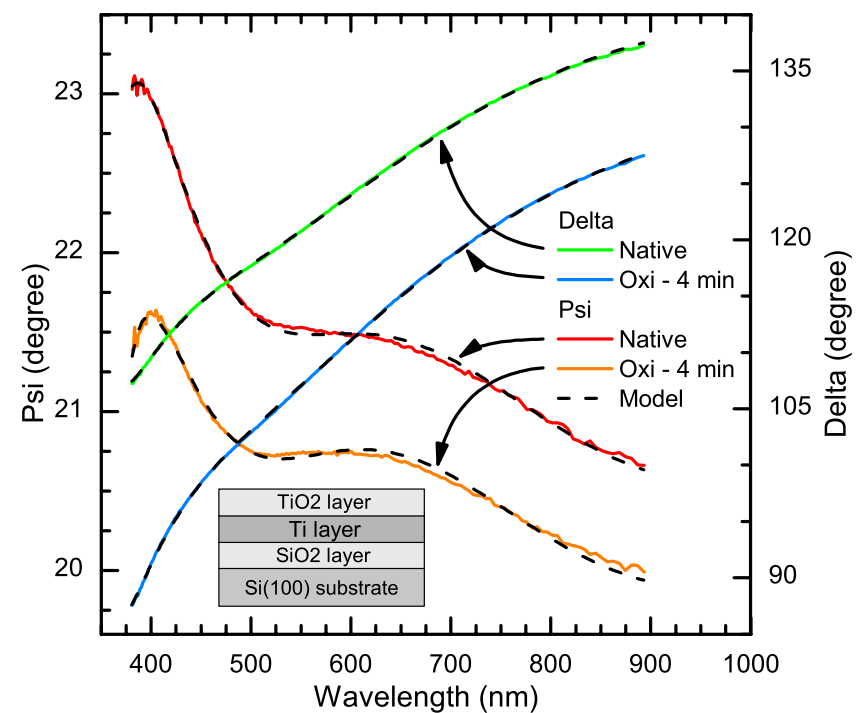

FIG. 1. (Color online) Psi-delta measurement data from two samples of series B. The first sample was measured after Ti evaporation (with exposure to air, thus with native oxide) and the second was oxidized in plasma for 4 min. The dashed lines give the best-match model data. The inset shows the layer stack of the sample which was exactly the same as the sample model stack.

multiple samples with thicknesses far apart may decrease the quality of the resulting best-matched data. Therefore, the dielectric functions of Ti were assumed constant in a range of a few nanometers and only series of samples with thicknesses within this range were fit together. For example, samples that started with the same Ti thickness (e.g., $20 \mathrm{~nm}$ ) and that were oxidized for different durations were fit together since the Ti thickness variation was relatively small.

The sample model as it was used to get the data mentioned in Sec. IV is shown in the inset of Fig. 1. No interface or roughness layer was used in the model because it did not improve significantly the quality of the fit. Also, results obtained with the interface and/or roughness layers were not consistent. Figure 1 shows data for two typical samples, one with native oxide only and one that was oxidized in $\mathrm{O}_{2}$ plasma for $4 \mathrm{~min}$. The best-match model data are also shown and can be seen to fit well on the experimental data, confirming the quality of our model. Notice that the higher level of noise at both ends of the spectra may be caused by the higher absorption of $\mathrm{Ti}$ in these regions but did not seem to affect the quality of the fit significantly. In Fig. 2, a series of uniqueness tests is shown for three samples oxidized in plasma for 1, 2, and $4 \mathrm{~min}$. In these tests, the thickness of the $\mathrm{TiO}_{2}$ layer is varied over a range of values while the other parameters are fit to find the best match to the experimental data. For these three samples the uniqueness range, as defined by Hilfiker et al., ${ }^{12}$ is of $\sim 0.3 \mathrm{~nm}$, which also confirms the quality and sensitivity of this model to the thickness of the layers. Similar uniqueness ranges were obtained for the Ti layer thickness. 


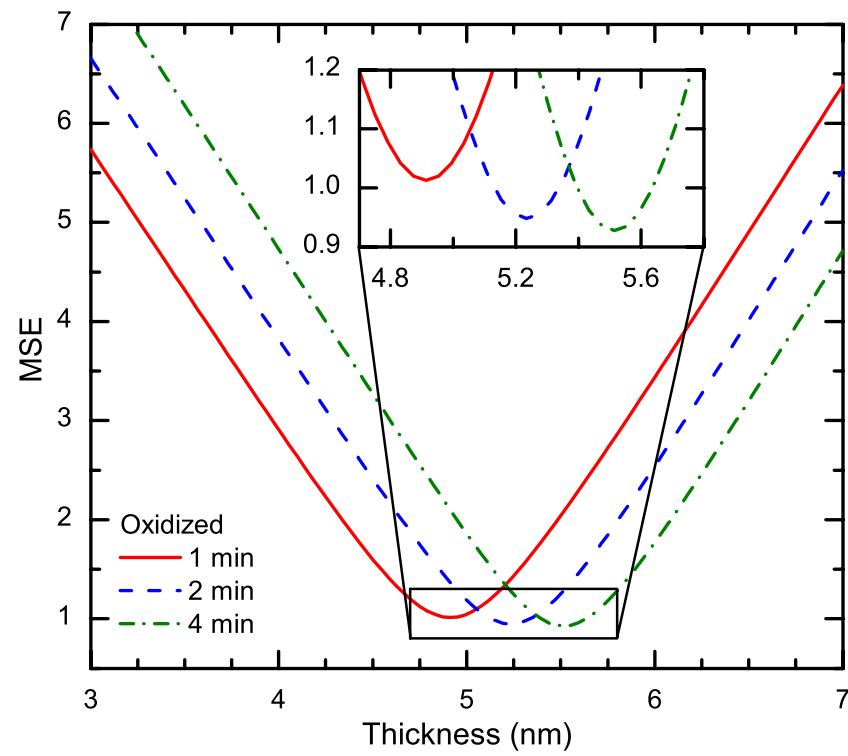

Fig. 2. (Color online) Thickness-uniqueness test data for part of series B. Samples were oxidized in plasma for 1-10 min. The curves give the MSE for each sample varying the thickness of the Ti layer in a predefined range and regressing the other parameters to find the best match to the experimental data. The inset shows a zoom on the minimum of each curve.

\section{RESULTS AND DISCUSSION}

\section{A. Validation of the ellipsometric model}

To validate the ellipsometric model, another measurement technique had to be used to determine the thickness of the Ti and $\mathrm{TiO}_{2}$ layers independently. Profilometry is a convenient method to measure steps with nanometric precision. However, since unstructured samples are used, patterning had to be performed. To achieve vertical steps in the Ti layer, patterns were made in samples of series B using a lift-off technique. The steps were then measured by profilometry and gave an average of $22.0 \mathrm{~nm}$ thickness for $\mathrm{Ti}$ and $\mathrm{TiO}_{2}$ together. Ellipsometry measurements on three samples with identical Ti film gave an average thickness of $\sim 22.9 \mathrm{~nm}$. This validates the accuracy of the ellipsometric model within approximately $1 \mathrm{~nm}$.

XPS measurements were taken on four different samples of series $\mathrm{B}$ to verify the stoichiometry of the $\mathrm{TiO}_{2}$. The main Ti peak $\left(\mathrm{Ti} 2 p_{3 / 2}\right)$ for a sample with native oxide only and one oxidized at $30 \mathrm{~W}$ for $1.5 \mathrm{~min}$ are shown in Fig. 3. The secondary peak (Ti $2 p_{1 / 2}$ ) and satellites are identified in the figure as well. As mentioned by McCafferty and Wightman, ${ }^{17}$ the main peak at $459.0 \pm 0.1 \mathrm{eV}$ has been identified to $\mathrm{Ti}^{4+}$, related to the stoichiometric $\mathrm{TiO}_{2}$. The arrows indicate the energies of the substoichiometric $\mathrm{Ti}_{2} \mathrm{O}_{3}\left(\mathrm{Ti}^{3+}\right)$ and $\mathrm{TiO}\left(\mathrm{Ti}^{2+}\right)$ oxides as well as the metallic titanium peaks. We see that the small amount of these suboxides quickly disappear after just $1.5 \mathrm{~min}$ of low power plasma oxidation. There is still a small Ti metallic peak, but it can be attributed to the fact that the $\mathrm{TiO}_{2}$ layer is still thin enough to let some metallic Ti electrons come through the layer. This completely disappears when increasing the plasma time. The XPS measurements thus show that the oxide created by the $\mathrm{O}_{2}$ plasma

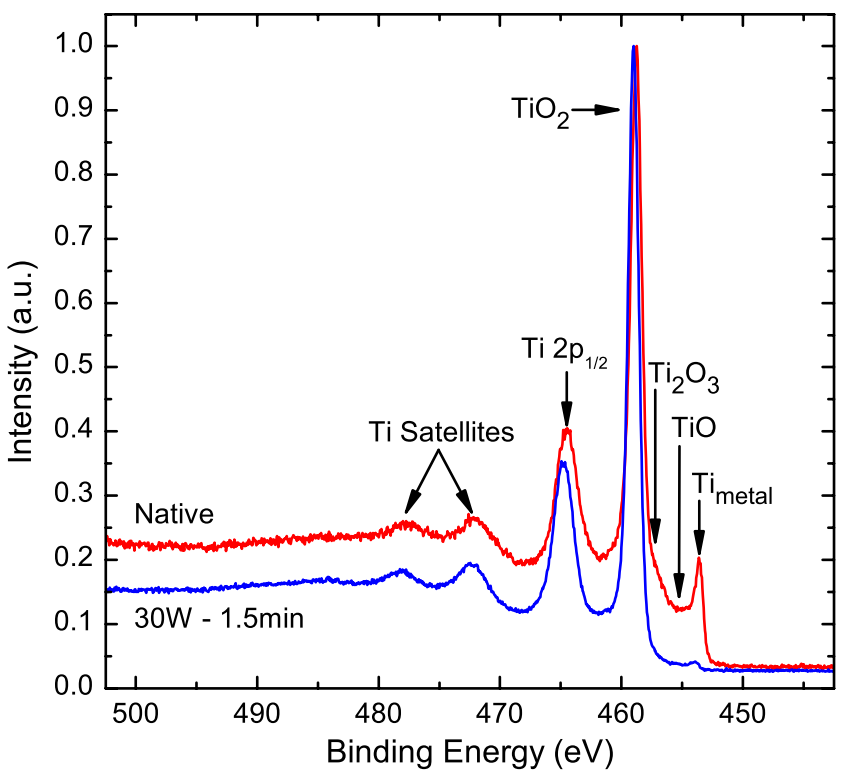

FIG. 3. (Color online) XPS spectra of samples of series B showing the main Ti peak. The curves have been calibrated with respect to the $\mathrm{C} 1 \mathrm{~s}$ peak at $285 \mathrm{eV}$ and normalized for comparison.

is fully stoichiometric even though the native oxide is not. Therefore, the ellipsometric data were fit with two different sets of the Cauchy parameters for the oxide layer, one for the samples before oxidation and the other for samples after oxidation.

\section{B. Characterization of a thin Ti layer oxidized by $\mathrm{O}_{2}$ plasma}

As already evoked, characterizing the growth of titanium oxide by plasma oxidation is crucial in order to control the size and capacitance of $\mathrm{TiO}_{2}$ potential barriers in the fabrication of SETs. ${ }^{14,15}$ Therefore, oxidation of series B was conducted as a function of time. An ICP reactor was used because it ensures good reproducibility, control, and process compatibility with standard microfabrication techniques. Errors on the ellipsometric measurements have been calculated with statistics on 13 identical samples before oxidation $(\bar{x} \pm 3 \sigma)$, which shows the variation on samples considered identical. It also incorporates nonidealities such as roughness and interface quality variations (since they were not explicitly modeled). An experimental error was also obtained from three identical samples oxidized in the same time, power, and pressure conditions.

Ellipsometric measurements on all samples used for the oxidation as a function of time and platen power give starting thicknesses of $1.3 \pm 0.4 \mathrm{~nm}$ for $\mathrm{TiO}_{2}$ and $21.2 \pm 1.2 \mathrm{~nm}$ for Ti. Figure 4 shows the gain in oxide thickness and the corresponding Ti loss after oxidation at $30 \mathrm{~W}$. The top curve shows a rapid increase of the oxide growth but seems to reach a maximum value of about $4.1 \pm 0.4 \mathrm{~nm}$ after $5-10$ min. Added to the native oxide present before oxidation, the total oxide thickness reaches $\sim 5.4 \pm 0.4 \mathrm{~nm}$. The bottom curve also saturates at about $-3.1 \pm 1.2$ thus leaving $18.1 \pm 1.2 \mathrm{~nm}$ of Ti after oxidation. All other measurement 


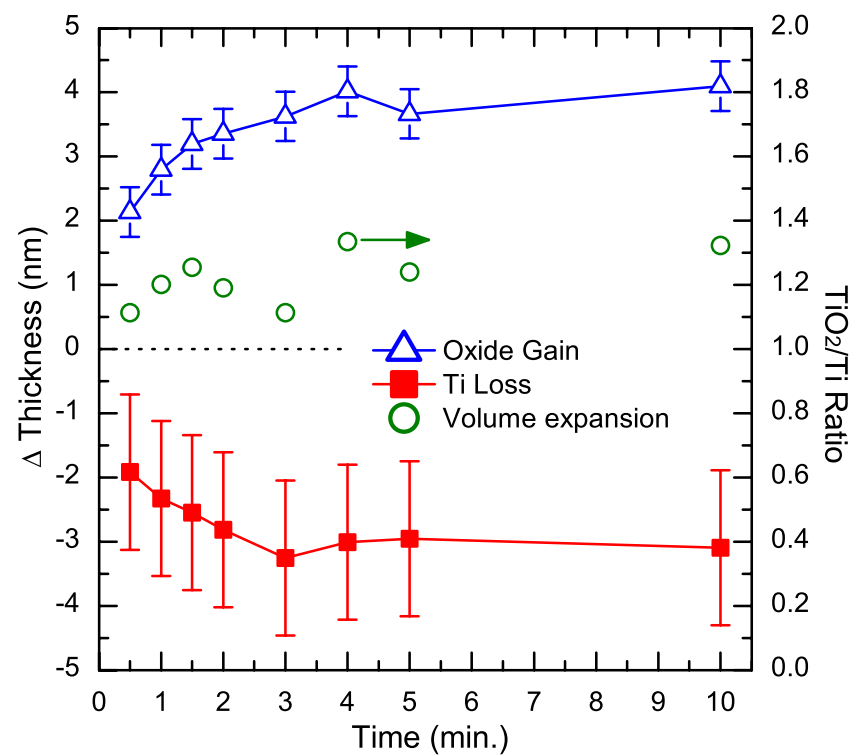

FIG. 4. (Color online) Ti loss and $\mathrm{TiO}_{2}$ gain in thickness as a function of plasma oxidation time for a platen power of $30 \mathrm{~W}$. The right axis shows the volume expansion ratio calculated from the oxidation data.

series also exhibited this saturation behavior for longer oxidation times and other starting Ti thicknesses (not shown). From these data, we can also calculate an approximate value for the volume expansion of $\mathrm{TiO}_{2}$ versus Ti. It is simply given by the absolute value of the ratio of the $\mathrm{TiO}_{2}$ gain and the Ti loss for each sample. The volume expansion shown in Fig. 4 is relatively stable between 1.1 and 1.4.

In order to better control and standardize a process it is often more practical to vary a parameter other than time. In this manner, the plateau appearing for high oxidation times enables a variation of another parameter such as the acceleration of the plasma ions toward the sample without worrying about oxidation time variations. Therefore, platen power was varied from 15 to $240 \mathrm{~W}$ with a constant oxidation time of $10 \mathrm{~min}$. Data shown on Fig. 5 also reveal a saturation behavior for the oxide creation curve. The increase in thickness of the oxide at $240 \mathrm{~W}$ is of $6.3 \pm 0.4 \mathrm{~nm}$ which is $2.2 \pm 0.8 \mathrm{~nm}$ higher than that of $15 \mathrm{~W}$ and gives an overall maximum oxide thickness of $7.6 \pm 0.4 \mathrm{~nm}$ for the plasma conditions used. This enables an easy configuration for future oxidation steps in the fabrication process of SET devices. The Ti curve is more linear showing continuous decrease at higher powers. The volume expansion seems to follow the same trend with a roughly linear decrease at higher powers. The decrease is from $\sim 1.3$ to less than 0.8 in the range used.

Differences in the oxidation rate have been observed and attributed to a routinely observed and measured He leak in the ICP chamber. This helium is used as a cooling agent to keep the sample at $20{ }^{\circ} \mathrm{C}$ but the seal between the plasma chamber and the He back cooling flow is done with the sample holder and varies from run to run. Considering this, part of the $\mathrm{O}_{2}$ plasma is composed of the leaked He which affects the oxidation dynamics. An estimate of the process

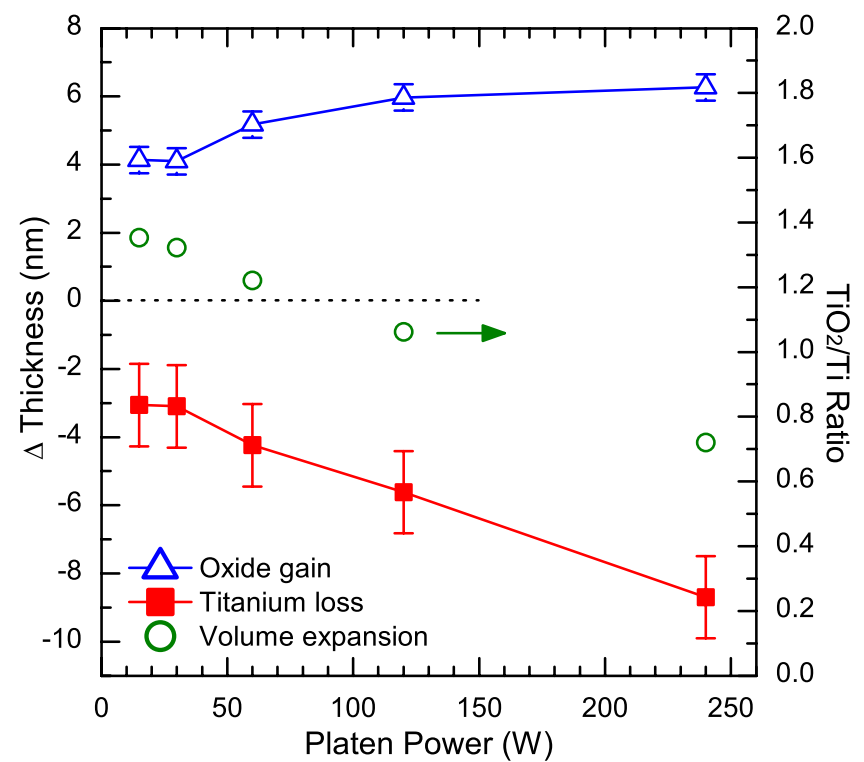

FIG. 5. (Color online) $\mathrm{Ti}$ loss and $\mathrm{TiO}_{2}$ gain in thickness as a function of the platen power for $10 \mathrm{~min}$ of oxidation time. This power is proportional to the $V_{p-p}$ potential applied on the sample holder. The right axis shows the volume expansion ratio calculated from the oxidation data.

variation caused by this effect is therefore included in the error bars shown in Figs. 4 and 5. It is expected that the volume expansion would not vary since the stoichiometry of the oxide is stable with any plasma time or power, which is seen in Fig. 4 and confirmed by the previously mentioned XPS measurements. Increasing the oxidation time then does not increase the penetration depth of of the ions at a fixed platen power. However, the linear decrease of both titanium thickness and volume expansion can be explained by the increased sputtering that occurs at higher powers. Indeed, with higher power comes higher acceleration and a deeper penetration of the ions accompanied by a mechanical etching of the surface. The surface is therefore oxidized and at the same time etched. The two phenomena reach an equilibrium consuming more $\mathrm{Ti}$ for the same $\mathrm{TiO}_{2}$ thickness created in the end. It is therefore normal to see the volume expansion dropping under unitary values. The variation of time and power thus shows a relatively good control on the oxide thickness created, although the decrease in Ti thickness has to be kept in mind for the calculation of feature sizes in actual devices.

\section{Characterization of a thin Ti layer processed by chemical mechanical planarization}

CMP is a key process used in the nanodamascene method for the fabrication of single electron devices. ${ }^{14,15}$ It is important to characterize the material removal rate (MRR) of this process since a nanometer control of the planarization is required for the fabrication of room-temperature single electron devices. This was carried out with samples of series A and by planarization intervals of 15-60 s. Measurements taken on two samples are combined in Fig. 6. The filled squares show a roughly linear dependence of material thick- 


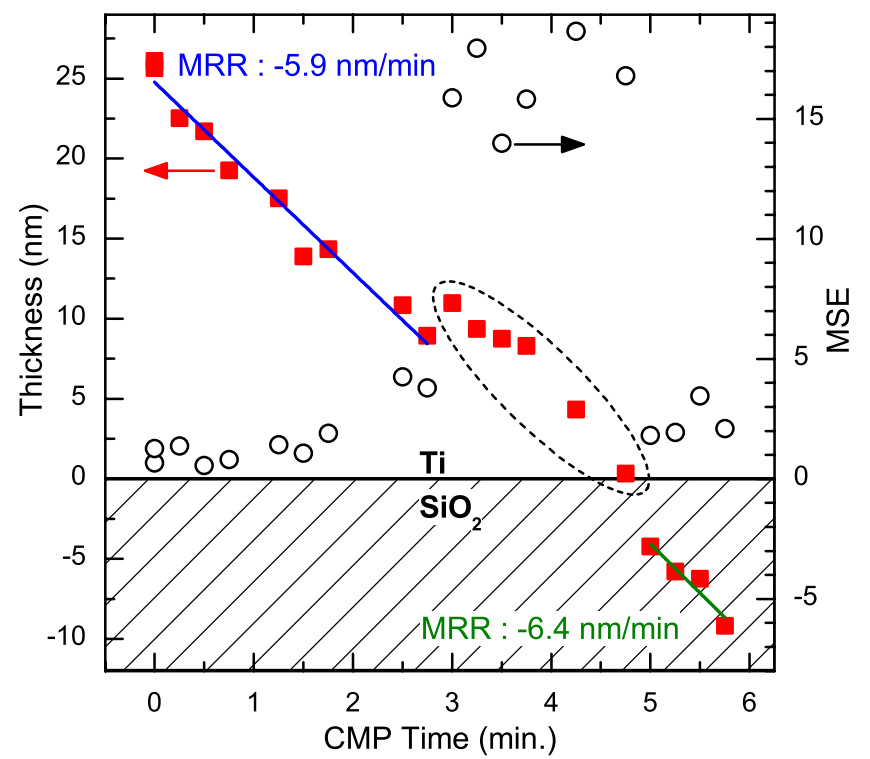

FIG. 6. (Color online) Sum of $\mathrm{Ti}$ and $\mathrm{TiO}_{2}$ thicknesses (left) and MSE of the ellipsometry fit (right) as a function of the chemical mechanical planarization time. Hatched region represents the $\mathrm{SiO}_{2}$ layer. Fits provide the MRR while the MSE enables one to evaluate the quality of each ellipsometry fit.

ness as a function of CMP time. The top left linear fit gives a $\mathrm{Ti}-\mathrm{TiO}_{2} \mathrm{MRR}$ of $5.9 \mathrm{~nm} / \mathrm{min}$. The negative thicknesses at longer planarization times mean that $\mathrm{Ti}$ and $\mathrm{TiO}_{2}$ have been entirely polished away and that $\mathrm{SiO}_{2}$ is being polished, here, with a MRR of $6.4 \mathrm{~nm} / \mathrm{min}$.

Since the thickness variations are too important to use MSA in this particular case, each measurement is fit individually and the MSE is used to estimate the accuracy of the fits. The MSE is below 5 at the beginning and then suddenly goes up to more than 10 when approaching the surface. When all the $\mathrm{Ti}$ and $\mathrm{TiO}_{2}$ are removed, $\mathrm{SiO}_{2}$ is being polished and the MSE goes back to values lower than 5. The high values near the $\mathrm{Ti} / \mathrm{SiO}_{2}$ interface can be explained by the nonuniformity of the Ti film at these thicknesses. Indeed, the nanoparticles mechanically removing the material leave behind a surface composed of scattered patches of $\mathrm{Ti}$ and $\mathrm{SiO}_{2}$. Since the model assumes a perfectly flat surface, it is easy to see that the encircled data in Fig. 6 are not accurate and are therefore not used to determine the MRR. This was confirmed by observation of different colors on the surface of the sample between the process steps. Also, some ellipsometric measurements of series B with 5- and 10-nm-thick Ti have been made and showed high MSE and inconsistent thicknesses, confirming that very thin layers of Ti are more difficult to measure with this method.

\section{SUMMARY}

Ellipsometry measurements taken on samples treated with plasma oxidation and chemical mechanical planarization show that it is possible to develop a reliable model and characterize the thickness of titanium and titanium oxide thin films and layers. However, great care must be taken in the development of the model because the absorption of light by
Ti or other metals causing a change in the dielectric functions with thickness of thin films decreases the quality of measurements and fits. MSA has been shown to greatly reduce this problem. It was also shown that there is a plateau above $\sim 5 \mathrm{~min}$ of oxidation in the ICP reactor and that its height can be controlled by changing the acceleration voltage applied to the sample holder (platen power). This allows for a good control in the process of creating tunnel junctions in a SET. The ellipsometric model was then used to characterize the MRR of the CMP process. The technique is currently used to improve the chemical slurry in order to get a better MRR selectivity of Ti versus $\mathrm{SiO}_{2}$. This is necessary to prevent certain common CMP problems such as dishing and erosion of structures while planarizing. Finally, XPS measurements of oxidized samples showed that only $\mathrm{TiO}_{2}$ is formed when oxidizing in an oxygen plasma. Although ellipsometry was carried out ex situ in this study, it is, in principle, possible to use MSA with in situ ellipsometers placed either in an evaporator, in a plasma/thermal oxidizing chamber, or on a CMP setup.

\section{ACKNOWLEDGMENTS}

The authors would like to thank J. Hilfiker and T. Tiwald, engineers at J.A. Woollam Co., for their good advice and support in the development of the ellipsometric model. The authors are grateful to the Nanofabrication and Nanocharacterization Research Center staff and in particular to M.-J. Gour for her help with the ICP manipulations. The authors also thank S. Blais from the Centre for Characterization of Materials for having acquired and analyzed XPS data. The authors finally thank NSERC for the funding of this work.

${ }^{1}$ L. L. Chang, L. Esaki, and R. Tsu, Appl. Phys. Lett. 24, 593 (1974).

${ }^{2}$ J. R. Tucker, C. Wang, and P. S. Carney, Appl. Phys. Lett. 65, 618 (1994).

${ }^{3}$ E. S. Snow, P. M. Campbell, R. W. Rendell, F. A. Buot, D. Park, C. R. K. Marrian, and R. Magno, Appl. Phys. Lett. 72, 3071 (1998).

${ }^{4}$ W. J. Gallagher and S. S. P. Parkin, IBM J. Res. Dev. 50, 5 (2006).

${ }^{5}$ J. M. Daughton, Thin Solid Films 216, 162 (1992).

${ }^{6}$ S. van Dijken, X. Jiang, and S. S. P. Parkin, Phys. Rev. B 66, 094417 (2002).

${ }^{7}$ S. van Dijken, X. Jiang, and S. S. P. Parkin, Appl. Phys. Lett. 80, 3364 (2002).

${ }^{8}$ K. Likharev and V. Semenov, IEEE Trans. Appl. Supercond. 1, 3 (1991). ${ }^{9}$ D. Koelle, R. Kleiner, F. Ludwig, E. Dantsker, and J. Clarke, Rev. Mod. Phys. 71, 631 (1999).

${ }^{10}$ D. V. Averin and K. K. Likharev, J. Low Temp. Phys. 62, 345 (1986).

${ }^{11}$ R. V. Patil and G. B. Kale, J. Nucl. Mater. 230, 57 (1996).

${ }^{12}$ J. N. Hilfiker, N. Singh, T. Tiwald, D. Convey, S. M. Smith, J. H. Baker, and H. G. Tompkins, Thin Solid Films 516, 7979 (2008).

${ }^{13} \mathrm{H}$. Fujiwara, Spectroscopic Ellipsometry Principles and Applications (Wiley, West Sussex, England, 2007).

${ }^{14}$ C. Dubuc, J. Beauvais, and D. Drouin, IEEE Trans. Nanotechnol. 7, 68 (2008).

${ }^{15}$ A. Beaumont, C. Dubuc, J. Beauvais, and D. Drouin, IEEE Electron Device Lett. 30, 766 (2009).

${ }^{16}$ CompleteEASETM Data Analysis Manual, Version 3.65, J. A. Woollam Co., Inc, 2008.

${ }^{17}$ E. McCafferty and J. P. Wightman, Appl. Surf. Sci. 143, 92 (1999).

${ }^{18}$ C. Herzinger, B. Johs, W. McGahan, J. A. Woollam, and W. Paulson, J. Appl. Phys. 83, 3323 (1998).

${ }^{19}$ Handbook of Ellipsometry, edited by H. G. Tompkins and E. A. Irene (William Andrew, Norwich, NY, 2005). 